\title{
Le cheminot en Italie. Image et culture
}

The Italian railwaymen's image and culture

\section{Stefano Maggi}

Traducteur : Walter Gennari

\section{OpenEdition \\ Journals}

Édition électronique

URL : https://journals.openedition.org/rhcf/165

DOI : 10.4000/rhcf.165

Éditeur

Rails \& histoire

Édition imprimée

Date de publication : 1 décembre 2007

Pagination : 36-37

ISSN : 0996-9403

\section{Référence électronique}

Stefano Maggi, "Le cheminot en Italie. Image et culture », Revue d'histoire des chemins de fer [En ligne], 36-37 | 2007, mis en ligne le 12 mai 2011, consulté le 22 avril 2022. URL : http:// journals.openedition.org/rhcf/165; DOI : https://doi.org/10.4000/rhcf.165 


\title{
Le cheminot en Italie. Image et culture
}

Texte traduit de l'italien par Walter Gennari

\author{
Stefano Maggi \\ Enseignant-chercheur à l'université de Sienne
}

Les chemins de fer en Italie. Du Risorgimento à l'étatisation

À cause du retard accusé par le développement économique du pays et de la lenteur du processus d'unification nationale, achevé en mars 1861 (à l'exception de Venise et de Rome), la réalisation d'un réseau ferroviaire italien commença seulement dans les années 1870, même si certains petits réseaux - d'une longueur totale de 2189 kilomètres - existaient déjà au Piémont, en Lombardie, en Toscane, dans les États de l'Église et au Royaume des Deux-Siciles.

Pendant toute la période du Risorgimento les chemins de fer constituèrent le principal symbole de l'unité nationale, car ils apparaissaient comme un lien entre les territoires de la Péninsule italienne qui était gouvernée par différents souverains. Selon le comte Cavour, qui fut le principal auteur de l'unification, les chemins de fer auraient favorisé «l'esprit de nation en Italie $»^{1}$.

Avant 1865 la gestion de ces lignes éparses n'était pas clairement définie. On trouvait aussi bien des lignes gérées par des sociétés privées que des chemins de fer d'État, surtout dans le Piémont. En 1865 on décida de confier à trois sociétés privées la construction des lignes et l'exploitation : Strade Ferrate Alta Italia, Strade Ferrate Romane, Strade Ferrate Meridionali, auxquelles vinrent se joindre la société Vittorio Emanuele pour les chemins de fer de Calabre et de Sicile et la Compagnia Reale Sarda pour les chemins de Sardaigne. Toutefois ce système entra en crise et l'Etat dut payer des sommes considérables pour racheter les chemins de fer et équilibrer le bilan des sociétés déficitaires.

1- Article de La Revue Nouvelle, in Camillo Cavour, Le Strade ferrate in Italia, édition de A. Salvestrini, Florence, La Nuova Italia, 1976, p. 61. 
Un long débat commença pour déterminer un nouveau mode de gestion. À partir de 1885 l'exploitation fut confiée à trois grandes sociétés tandis que la propriété des lignes et des installations restait à l'État. La péninsule italienne fut partagée verticalement, dans le but de développer le trafic entre le Nord et le Sud et pour surmonter les dissensions entre les deux parties du pays. Le réseau oriental fut confié à la Rete Adriatica, qui exploitait $4379 \mathrm{~km}$ de lignes au total, tandis que le réseau occidental, long de $4171 \mathrm{~km}$, fut attribué à la Rete Mediterranea ; la Rete Sicula devait gérer $1100 \mathrm{~km}$ de lignes en Sicile. La durée des concessions de 1885 était au maximum de 60 ans, répartie en trois périodes de 20 ans chacune.

Ce système donna d'aussi mauvais résultats que le précédent, dans la mesure où l'État fut forcé d'intervenir financièrement, si bien que après un débat très violent, on en arriva en 1905, avant la plupart des autres États européens, à nationaliser $14700 \mathrm{~km}$ de chemins de fer, soit la majorité du réseau : seuls $2700 \mathrm{~km}$ furent confiés à de petites compagnies locales ${ }^{2}$.

L'entreprise ferroviaire d'État (Ferrovie dello Stato) fut ainsi fondée et placée sous la tutelle du ministre des Travaux publics ; son conseil d'administration avait néanmoins une liberté d'intervention en ce qui concernait les choix de gestion. Ce conseil était en fait dominé par le directeur général de l'entreprise, à la tête de laquelle le leader politique Giovanni Giolitti mit l'ingénieur Riccardo Bianchi. En 1905 il y avait en Italie environ 118000 cheminots.

\section{Travail, politique et syndicat ferroviaire}

La recherche sur les cheminots a été jusqu'à ce jour assez fragmentaire en Italie. Les travaux existants s'intéressent essentiellement aux premières formes de syndicalisme et se concentrent sur la période antérieure au fascisme. Quant aux recherches locales sur les ouvriers qui ont construit les voies ferrées, sur l'origine géographique et sociale des cheminots, sur leur rapport à la politique et surtout sur les métiers et sur l'identité professionnelle, elles restent aujourd'hui fort incomplètes.

De telles recherches pourraient pourtant permettre de comprendre le mouvement social dans des localités importantes qui, pendant de longues années, ont présenté des caractéristiques de « villes ferroviaires ». En effet, en Italie comme ailleurs en Europe se sont développés différents villes et bourgs ferroviaires au cours du XIX siècle (Bussoleno, Novi Ligure, Foligno, Chiusi, Orte). Il ne s'agissait pas de localités nées

2- Loi $\mathrm{n}^{\circ} 137$ du 20 avril 1905. A. Papa, Classe politica e intervento pubblico nell'età giolittiana. La narionalizzarione delle ferrovie, Naples, Guida, 1973. 
avec les chemins de fer, mais de villages qui ont rapidement crû grâce a l'existence des installations ferroviaires où les cheminots représentaient les travailleurs les plus nombreux. En outre les chemins de fer ont joué un rôle important dans le peuplement des territoires où n'existaient pas auparavant d'implantations urbaines, parce que les habitations et les usines se sont rassemblées autour des gares; elles ressemblèrent à des oasis, car elles représentaient de véritables avant-postes de la présence humaine.

Edmondo De Amicis, l'auteur du livre Cuore (cœur) ${ }^{3}$, écrit en 1908 dans son Voyage en Sicile:

«L'image des grandes rues pleines de monde de Palerme me suivit pendant tout le voyage que je fis de Palerme à Catane en traversant l'intérieur de l'île, laquelle me donna l'impression que cette région-là était plus triste et solitaire et que je ne la connaissais absolument pas. Mais quelle différence il y avait entre l'intérieur et les côtes! Les gares sont presque toutes perdues dans une grande solitude, comme si elles étaient des petites forteresses dans le désert ; elles sont loin des villes et des villages : quelquefois on n'arrive pas à les apercevoir, elles sont sur la cime des hauteurs rocheuses ${ }^{4}$.»

Une image très significative des cheminots italiens est liée à leur résidence dans les gares et dans les maisons cantonnières, loin de toutes les structures de la vie urbaine et souvent dans des territoires où sévissait le paludisme alors endémique dans la plupart des plaines de la péninsule et des îles. Comme le rappelait De Amicis, en Italie les villages se dressaient sur une hauteur et les gares furent en général construites dans les plaines environnantes. Les gares jouèrent un rôle dans le peuplement des nouveaux territoires, grâce aux travailleurs des chemins de fer. Les chemins de fer furent les premiers à utiliser la quinine comme médicament et à expérimenter la prophylaxie contre le paludisme, par l'usage des moustiquaires sur toutes les fenêtres. De cette façon la maladie baissa chez les cheminots, de $70 \%$ de l'effectif à la fin du XIX siècle à 11 \% en 1907. En 1908 l'épidémie de paludisme s'étendit encore beaucoup, mais « resta toujours légère le long des lignes ferroviaires et il y avait un grand contraste entre cette population et la population rurale qui l'entoure $»^{5}$. (Fig. 1.)

3- Edmondo De Amicis, Cuore, 1886, raconte l'histoire d'une classe de l'école primaire, dont un des enfants est fils d'un mécanicien, après l'unification du royaume d'Italie.

4- Edmondo De Amicis, Viaggio in Sicilia. E note per chi viaggia oggidì, Veronelli, 1994, édition originale 1908 .

5- Angelo Celli, Manuale dell'ufficiale sanitario. Corso di perfezionamento, Rome, Società editrice Dante Alighieri, s.d., p. 276-277. 
Dans un livre de souvenirs intitulé Fils de cheminots le scénariste Ugo Pirro écrivait, à propos de son enfance au début du $\mathrm{XX}^{\mathrm{e}}$ siècle :

«Ces petites gares du paludisme, avec un trafic de voyageurs réduit, quand la nuit tombait fermaient comme n'importe quel magasin. Le cheminot et sa famille qui habitaient dans ces petites gares-là, comme Picerno, Contursi, Sicignano, perdues sous la pluie et dans la solitude, étaient ravitaillés par La Provida, un magasin roulant pour les cheminots, lequel passait chaque semaine, vendant des denrées alimentaires, des bougies stéariques, du pain rassis. Pour les bureaux des gares par contre, il apportait des imprimés, de la cire à cacheter, des zones, ces longues bandes de papier semblables à des serpentins de carnaval qui tournaient dans les appareils télégraphiques sur lesquels les ordres de service étaient transcrits ${ }^{6}$. »

Le service ferroviaire était l'un des premiers employeurs du monde du travail, que l'on prenne en considération la diversité des fonctions ou le nombre des heures travaillées : employés administratifs des directions et des bureaux (environ $10 \%$ du total), ingénieurs et autres spécialisations techniques de degré inférieur, employés chargés du tracé et de l'entretien des voies, du matériel roulant et des installations électriques. Les métiers liés à la circulation des trains comme les mécaniciens et les chauffeurs, le personnel de gare, les roulants (chefs de train, conducteurs, serre-frein) regroupaient plus de $60 \%$ des cheminots. À l'intérieur de ce pourcentage on pouvait trouver des groupes dont l'identité professionnelle était plus forte que d'autres et qui étaient aussi plus combatifs dans le champ syndical et politique.

En ce qui concerne ce dernier aspect, les cheminots ont représenté une des catégories les plus politisées et ce à partir de la dernière décennie du XIX ${ }^{\mathrm{e}}$ siècle, moment où l'idéologie socialiste se répandit parmi eux. Ils commencèrent à s'intéresser à la politique et au développement des premières organisations syndicales, grâce à l'acharnement de quelques pionniers. Le plus important parmi eux fut Cesare Pozzo, qui mourut tragiquement en 1898, après avoir apporté une contribution fondamentale à la naissance des unions des travailleurs qui se développèrent d'abord sous la forme de mutuelles. Ainsi la Società Nazionale di Mutuo Soccorso Cesare Pozzo, qui est encore aujourd'hui la principale société de secours mutuel d'Italie et est ouverte à tous les citoyens, est issue d'une ancienne société mutuelle de mécaniciens et chauffeurs fondée en $1877^{7}$.

6- Ugo Pirro, Figli di ferroviere, Palermo, Sellerio, 1999, p. 11-12.

7- Stefano Maggi, Il Tormento di un'idea. Vita e opera di Cesare Pozzo, Milan, Angeli, 1998. 
Avec le décollage industriel, les cheminots, à la fin du XIX siècle et surtout dans les premières années du $X^{e}$ siècle, ont représenté une base essentielle pour les forces politiques de gauche et le mouvement socialiste qui prenait alors son essor. Le premier cheminot député fut Quirino Nofri, élu au Parlement en 1897 sur les listes du parti socialiste italien, fondé en 1892.

Quelques années plus tard, l'écrivain Ugo Ojetti, dans son roman Mio figlio ferroivere (Mon fils le cheminot), raconte la vie d'un jeune bourgeois de retour de la Première Guerre mondiale. Son père, médecin, lui demande ce qu'il voudrait faire de sa vie et le jeune homme lui répond qu'il veut aller à Turin à l'école des mécaniciens. "Quels mécaniciens ? 》, lui demande son père, et lui de répondre : "Mon père, j’ai choisi ma profession. Je deviendrai cheminot... tu le sais, je suis socialiste ${ }^{8}$. »

Malgré la prédominance des forces de gauche, tous les courants politiques trouvèrent un terrain fertile chez les travailleurs des chemins de fer, surtout l'idéologie fasciste. Le mouvement de Mussolini se répandit parmi le personnel dirigeant, il produisit une fracture sociale à l'intérieur de l'entreprise jusqu'aux licenciements des années 1920, qui réduisirent l'effectif du personnel d'exécution de 235000 hommes en 1921 à 174000 en 1924. Une lettre de Benito Mussolini à un chef local, Roberto Farinacci, témoigne de l'importance des cheminots pour le mouvement fasciste :

"Cher Farinacci,

C'est à toi, qui es encore lié spirituellement aux cheminots fascistes, magnifique avant-garde des nos légions syndicales, que je confie la charge de faire parvenir mes salutations au congrès des cheminots fascistes de Lombardie. Je veux déclarer à travers toi et de la façon la plus directe que les cheminots fascistes ont pour une large part le mérite de ce qu'on peut nommer le renouveau ferroviaire de la Nation.

Aujourd'hui l'Italie a ses chemins de fer non seulement parce que la discipline nécessaire de la société humaine est partout rétablie, mais parce que l'Italie a des cheminots, c'est-à-dire des agents laborieux, intelligents, ordonnés, fidèles aux intérêts de la Patrie...

La Nation peut compter sur les cheminots fascistes; qu'ils se rendent pleinement compte que leur rôle est très important et extrêmement délicat parce que les chemins de fer représentent le système nerveux pour les organismes des nations modernes ${ }^{9}$. »

8- Ugo Ojetti, Mio Figlio ferroviere, Milan, Treves, 1922, p. 30.

9- Paolo Orano, I Ferrovieri per la rivoluzione delle camicie nere, Rome, Casa Editrice Pinciana, 1934, p. 14-15. 
Les cheminots italiens ont été toujours très politisés. Au-delà de leur puissant syndicat, au cours des années ils ont fourni des dirigeants aux partis politiques et aux institutions. Dans le Parlement élu au mois de mai 1921 siégeaient dix cheminots, six encore dans l'assemblée constituante élue en 1946. En effet leur degré d'instruction était assez élevé et l'alphabétisation répandue parmi eux, dans une société où l'analphabétisme dominait encore. En outre, le fait qu'ils travaillaient pour des entreprises privées rapprochait les cheminots de la mentalité ouvrière et les différenciait des employés de la bureaucratie ministérielle malgré leur tutelle commune. C'est à cause de cette mentalité particulière que les syndicats et les idéologies politiques avaient trouvé chez les cheminots un fort enracinement qui continua après l'étatisation réalisée en 1905 avec la création des Ferrovie dello Stato.

Les caractères du travail cheminot, à savoir une grande responsabilité personnelle, l'esprit d'équipe, le dévouement au métier, permirent en outre le succès d'un syndicat fort, lequel devint au début du XX siècle un modèle pour les autres organisations des travailleurs, surtout après la fondation en mai 1907 de l'organisation unitaire : le Syndicat des cheminots italiens (Sindacato ferrovieri italiani - SFI). Il recruta jusqu'environ $80 \%$ des cheminots, un taux très élevé par rapport à la moyenne italienne.

L'histoire même des grèves fut liée au transport ferroviaire, étant donné la combativité des cheminots qui les distinguait dans le monde du travail : on leur doit, au mois d'avril 1905, la première grève catégorielle nationale. Toutefois les premières grèves commencèrent pendant les années 1880, et les sanctions prévues par le code pénal en vigueur dès 1859 - la peine de réclusion pour la grève « sans une cause raisonnable »-plurent sur les cheminots.

Le nouveau code pénal de 1889 libéralisa théoriquement la grève, à condition qu'elle ne soit pas menée avec « violence et menace », mais on continua à interdire les grèves des cheminots parce qu'elles interrompaient l'exécution du service public national le plus important ${ }^{10}$.

Malgré les changements techniques qui se sont produits au cours d'un siècle et demi, l'identité professionnelle et la combativité politique et syndicale des cheminots sont restées presque intactes jusqu’à la restructuration qui a débuté au milieu des années 1980 et a bouleversé le travail et les habitudes de vie des cheminots mais aussi leur image pour le public.

10- Lorenzo Gaeta, «Materiali per una storia dello sciopero nei servizi pubblici : il periodo liberale », Lavoro e diritto, $\mathrm{n}^{\circ} 1$ (janvier 1989), p. 134-135. 


\section{La profession du cheminot : image et mentalité}

Les cheminots se répartissaient entre de nombreux métiers très différents par leur contenu professionnel, leur formation, leur rétribution et la qualité de vie qui leur était attachée. En 1923 le service était organisé en plus de 100 différents métiers qui se distinguaient aussi par l'origine sociale de leurs titulaires : tandis que les employés et les « chefs » des différents secteurs appartenaient en général à la petite bourgeoisie urbaine, les cheminots de statut ouvrier venaient en grande partie des boutiques d'artisans, où les travailleurs apprenaient un métier utile pour le monde du travail ferroviaire. Vers la fin du XIX siècle, grâce au progrès de la formation, les ouvriers spécialisés furent choisis parmi les diplômés des écoles techniques.

Le personnel le plus nombreux, avec un métier ferroviaire spécifique, comme les ouvriers, les gardes-frein, les hommes chargés des manœuvres, les aiguilleurs, venaient pour leur majeure partie des petites villes et de la campagne. Cette origine prédomine pendant les années de la fin du XIX ${ }^{\mathrm{e}}$ siècle et au début du XX ${ }^{\mathrm{e}}$ siècle mais également pendant le deuxième après-guerre, lorsqu'à cause du dépeuplement des campagnes les travailleurs se déversèrent en masse dans l'industrie et dans le tertiaire. En ce qui concerne cette dernière période, il faut aussi noter l'apport du sud de l'Italie, où il n'y avait pas d'industrie ; les cheminots qui en étaient issus suivaient la tendance générale des fonctionnaires publics italiens, qui venaient du Midi.

Il semble qu'être issu des petites villes de province et surtout des villages soit une caractéristique de longue date du cheminot italien aux échelons les plus bas, sans doute parce que travailler aux chemins de fer leur semblait plus attrayant que dans leur lieu d'origine, tandis qu'en ville les usines et les bureaux des administrations publiques et des banques exerçaient sur les jeunes gens une attraction aussi forte que les chemins de fer. Le quotidien communiste L'Unità écrivait en 1977 :

«Autrefois, il y a 30 ou 40 ans, on vous appelait avec admiration et envie orovieri (chemin-or), l'arma azzurra ('arme d'azur) de la classe ouvrière. »

« Il est vrai, surtout en province nous étions de bons partis pour les jeunes filles comme il faut, nous étions révérés et recherchés par les parents des jeunes filles célibataires ${ }^{11}$. »

11- L’Unità, 22 décembre 1977. 
Malgré cette grande diversité professionnelle et cette stratification sociale, la cohésion de la corporation était réelle ; sa solidité tenait à la fierté commune à tous de travailler au chemin de fer et de leur attachement à leur métier ; ce dernier aspect était considéré comme ayant une importance fondamentale pour le développement de l'économie et de la société.

En Italie un tel sentiment était plus marqué encore à cause du retard de l'industrialisation. Pour se faire une idée de la mentalité des cheminots et de leur force de pression à cette époque, il faut lire ces phrases saisissantes que le juriste Giuseppe Cimbali écrivait dans le premier traité complet de droit administratif italien :

«Le personnel ferroviaire, qui est conscient d'être un organe du mouvement central et miraculeux de la vie moderne, s'estime au-dessus de tous et estime avoir le droit, pour cette raison, à toutes sortes de tolérances et de privilèges. Comme il participe à la violence qui s'échappe des locomotives à vapeur et des dynamos électriques, il est intolérant à l'égard de tout joug et à toute discipline. Il montre une sensibilité suprême, ayant l'habitude de faire de vertigineuses courses, et dédaigne les obligations des règles communes...

Se déclarer cheminot c'est comme répéter l'ancienne et orgueilleuse diction civis romanus sum. Il s'agit d'une puissance, qui est nouvelle dans l'histoire et devant laquelle tout le monde, même l'État, doit baisser les armes et capituler ${ }^{12}$.»

La caractéristique la plus spécifique du service des chemins de fer était la grande responsabilité personnelle qu'assumaient les cheminots et qu'ils évoquaient sans cesse pour réclamer un salaire qui soit une juste compensation du risque judiciaire civil et pénal lié à leur activité. Ce sont des vies humaines qui dépendaient du mécanicien qui transportait des centaines de personnes, de l'aiguilleur, du garde-barrière, du cantonnier préposé à la surveillance des voies, de tous ceux qui devaient travailler de façon correcte pour les conserver. La responsabilité du cheminot était donc exceptionnelle ; une collaboration constante entre les travailleurs des différents services étaient indispensable pour éviter les catastrophes ferroviaires qui frappaient tant l'opinion publique. Les cheminots étaient souvent eux-mêmes victimes d'accidents et on leur attribuait le grand courage indispensable à l'exercice d'un emploi aussi périlleux.

12- Giuseppe Cimbali, « Le strade ferrate », in V.E. Orlando (dir.), Primo Trattato completo di diritto amministrativo italiano, Milan, Società editrice libraria, 1914, vol. VII, p. 936. 
Les catastrophes ferroviaires frappaient toujours beaucoup l'imagination populaire. Les accidents étaient dus au sort ou à des erreurs humaines : même les chanteurs ambulants, qui déclamaient sur les places des villes et des villages, se mirent à traiter ce sujet. L'importance des catastrophes ferroviaires dans l'imaginaire est attestée avec force par les œuvres d'Achille Beltrame, le célèbre dessinateur qui dès 1899 et pendant plus de 40 années illustra les actions d'éclat les plus importantes sur la couverture de La Domenica del Corriere qui était alors le magazine illustré le plus diffusé en Italie. Parmi les couvertures dédiées aux trains au cours des années, quelques-unes représentent en détail les accidents, avec des images apocalyptiques de déraillements, de collisions mettant en cause les hommes ou des animaux ; d'autres sont des scènes héroïques où des cheminots se sacrifient afin d'éviter un désastre ${ }^{13}$.

La possibilité d'une ascension progressive dans la hiérarchie était perçue positivement dans la représentation des cheminots. Elle était réelle au moins jusqu'aux niveaux intermédiaires, puisque le système d'avancement était fondé sur l'ancienneté et que les entreprises étaient très grandes, avec une considérable rotation du personnel due au fait que la perte des aptitudes physiques commandait l'affectation d'un grand nombre à des tâches moins lourdes. Un allumeur de chaudières pouvait terminer sa carrière comme mécanicien, un garde-frein comme chef de trains, un manœuvre comme responsable d'une halte ou même chef de gare (fig. 2).

Dans les classes modestes, un emploi aux chemins de fer était un objet de convoitise. Il promettait la sécurité de l'emploi et un salaire excellent, comparé à ce que les ouvriers en usine et même parfois les fonctionnaires pouvaient gagner. C'est ainsi que, au cours d'un procès mené contre Cesare Pozzo à cause de son activité politique et syndicale, une déclaration publique à propos de la rémunération des cheminots fit grand bruit : elle fut rapportée dans tous les journaux. En effet, un avocat s'était écrié : «il est bon de savoir qu'un mécanicien qui gagne 3500 lires par an se plaint de l'administration. Si tout le monde et les magistrats étaient si chanceux ${ }^{14}$ ! » Celui qui « entrait au chemin de fer », comme on disait avec orgueil, était d'ordinaire destiné à y rester malgré les dures conditions de travail qui lui étaient promises. Dans la société italienne du XIX siècle et de la première moitié du XX siècle, les cheminots jouissaient d'un singulier privilège : celui d'être la première « aristocratie ouvrière ».

13- Stefano Maggi, Le Ferrovie, Bologne, Il Mulino, 2003, p. 151-152.

14- «Processo Barazzuoli. Resoconto stenografico », Libertas, 30 mai 1891. 
Les cheminots étaient différents des autres travailleurs par leur revenu et par leur mentalité ; à l'exception des sites comme les ateliers de réparation du Matériel ou les vraies "villes ferroviaires » au sens propre du terme où ils étaient les plus nombreux, ils ne représentaient qu'une minorité des travailleurs de l'industrie, mais en même temps un secteur de pointe, pris pour modèle par les autres travailleurs. Même dans la vie quotidienne, les cheminots des quartiers ouvriers jouissaient d'une situation respectable et menaient une vie décente. Ils le faisaient savoir par leur façon de s'habiller, qui était une marque distinctive pour tous, très importante au XIX ${ }^{\mathrm{e}}$ siècle. Il faut rappeler aussi que les femmes des employés des chemins de fer engageaient quelquefois des femmes de ménage, surtout dans les villes du sud de l'Italie ${ }^{15}$.

Dans l'univers ferroviaire, divisé en une multitude de fonctions, le personnel qui contribuait à la circulation des trains représentait une classe particulière, qui vivait en général dans un univers homogène partagé seulement avec des camarades de même statut. Le matin, ces gens ne quittaient pas leur foyer pour « aller au travail » mais pour « prendre leurs fonctions ", une formule qui exprimait leur conviction d'exercer une profession importante qui les distinguait de l'ordinaire. Afin d'établir leur position sur l'échelle hiérarchique, les mécaniciens, les conducteurs, les hommes de manœuvre n'employaient pas le mot « ouvrier» : ils préféraient plutôt le terme « agent », qui indiquait mieux des compétences qui n'étaient pas purement manuelles. En outre dans le chemin de fer régnait un fort esprit militaire, bien identifiable à travers les uniformes et les coiffures galonnées.

La profession la plus caractéristique était sans doute celle du mécanicien, préposé à la locomotive, qui n'était pas simplement un conducteur mais un vrai «mécano» : au-delà de la conduite vapeur il devait être à même d'assurer l'entretien et de petites réparations. Dans un livre de mémoires publié en 1996, Franco Piccinelli, ancien présentateur de télévision et radio qui vécut près d'une voie ferrée dans le Piémont, écrit à propos du mécanicien :

«Afin de souligner l'aptitude professionnelle et la délicatesse du travail, le mécanicien avait le titre de maitre, un épithète unique parmi les cheminots. Grâce à son savoir-faire professionnel et à son ascendant universellement reconnu [...]

15- Travet, "Famiglie di ferrovieri», La Tecnica professionale, 1957 ; Daniele Jalla, «Perché mio papà era un ferroviere... Una famiglia operaia torinese dei primi del Novecento », Rivista di storia contemporanea, janvier 1980, p. 45. 
"Allez mon maitre !" disait l'homme de manœuvre au mécanicien pour lui faire avancer un peu le convoi : avec ce titre on voulait mettre en évidence une demande aimable, presque d'une faveur.

Le chauffeur s'adressait à son chef en lui démontrant par le titre de maitre son respect ; le mécanicien était son instructeur, son guide.

Les mécaniciens étaient aussi nommés affectueusement les visages noirs à cause de l'exposition prolongée à la suie... ${ }^{16}{ }$ ”

Les mécaniciens se distinguèrent toujours non seulement par une importante mobilisation syndicale et politique mais aussi par un esprit de corps plus marqué que dans les autres statuts. Au début du $\mathrm{XX}^{\mathrm{e}}$ siècle les mécaniciens et les chauffeurs avaient déjà leur syndicat ; ils avaient leurs propres écoles professionnelles à l'intérieur de l'entreprise, où ils obtenaient après un examen final les « abilitazioni », des certificats d'aptitude à la profession, lesquels les autorisaient à conduire différents types de locomotives ; ils avaient aussi leur propre journal, In Marcia!, fondé en novembre 1908, qui représentait un modèle pour la presse syndicale. La presse de l'entreprise est en effet révélatrice de l'image du cheminot, avec la publication mensuelle Voci della rotaia, fondée en janvier 1958 (fig. 3). Aujourd'hui cette presse représente une excellente source pour reconstituer les évolutions du travail et sa très forte culture. La presse ferroviaire locale s'accompagnait d'une bonne quantité de lieux de rencontre et d'institutions liées à la profession, comme les sociétés de secours mutuel déjà citées ou le Dopolavoro ferroviario (DLF), institution fondée par le fascisme en 1925 pour créer des centres récréatifs qui devaient organiser les loisirs des travailleurs ; il obtint dans le secteur ferroviaire une diffusion bien plus grande que les établissements analogues destinés aux autres corporations : près de chaque gare d'une certaine importance un dopolavoro fut édifié. En 1935 existaient 273 DLF locaux qui comptaient plus de 134000 sociétaires $^{17}$. Les DLF représentaient un lieu de rencontre important non seulement pour les cheminots mais aussi pour les habitants des environs. En outre les DLF les plus importants organisaient des excursions, des activités sportives et même des activités culturelles comme le concours littéraire Penna d'oro ferroviaria qui, chaque année, accordait un prix aux meilleurs récits écrits par les cheminots.

16- Franco Piccinelli, C'era una volta il treno, Turin, Sei, 1996, p. 67.

17- Mario Valdivieso, "Le attività sociali e assistenziali », in La Gestione di Stato delle ferrovie italiane (1905-1955). Monografie, Rome, 1956, p. 309. 


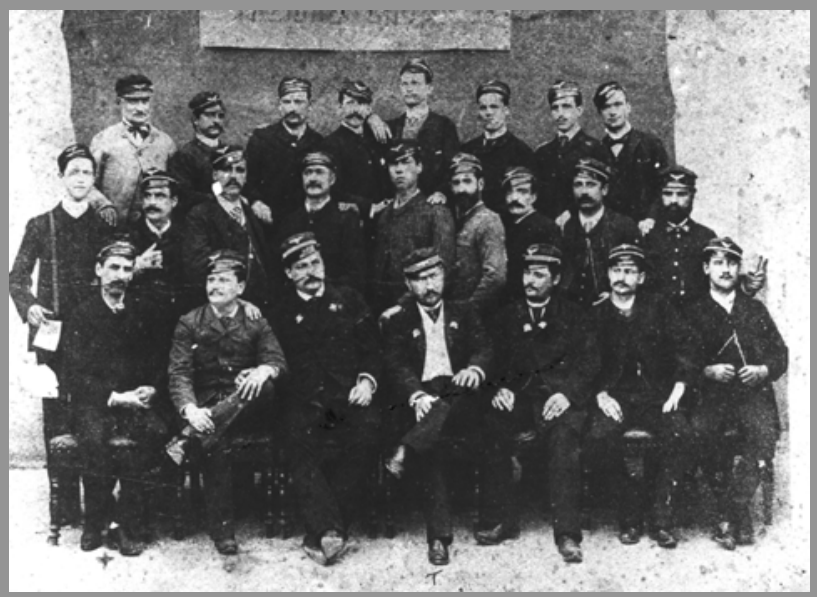

Cheminots à la gare de Grosseto, zone de paludisme, dans les premières années du XXe siècle.

Coll. Stefano Maggi.

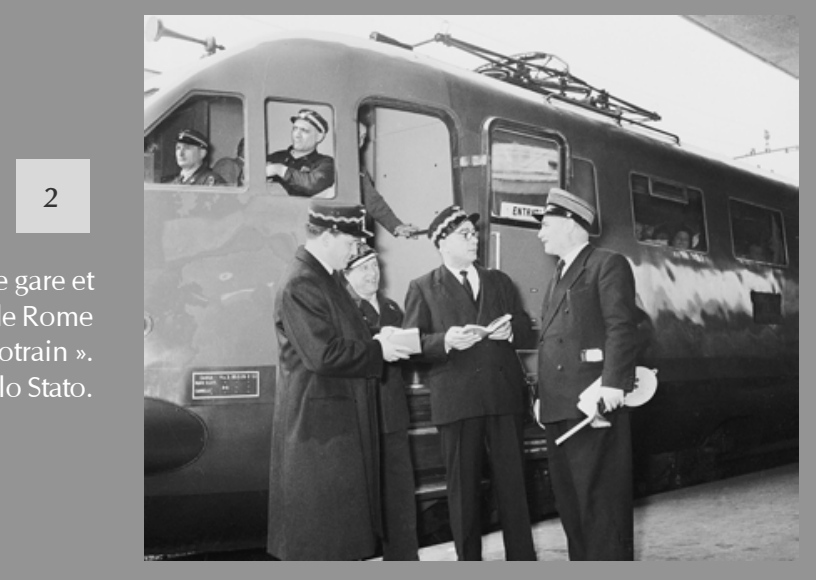

Chef de train, chef de gare et mécanicien à la gare de Rome Termini, devant un « électrotrain ».

Cl. Fototeca Ferrovie dello Stato.

École professionnelle des mécaniciens, Florence, 1953. Coll. Stefano Maggi.

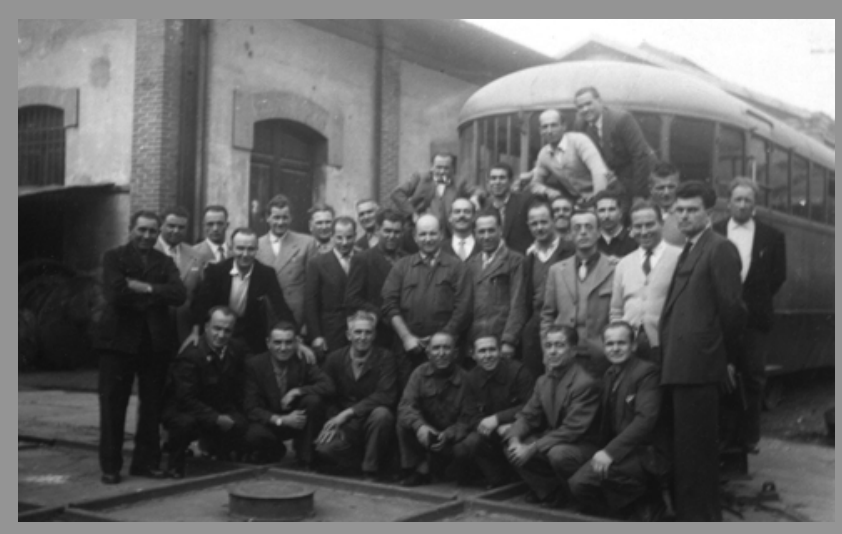




\section{Les restructurations et la fin de l'identité héritée}

Pendant les vingt premières années du $\mathrm{XX}^{\mathrm{e}}$ siècle, grâce à la diminution des heures de travail obtenue par les syndicats, le nombre des travailleurs augmenta et passa dans les chemins de fer de 102000 en 1900 à 235000 en 1921. La syndicalisation et la politisation des agents avaient atteint un degré sans précédent.

Une fois le fascisme au pouvoir, il agit d'une façon drastique. Le conseil d'administration des Chemins de fer de l'État fut dissout au mois de décembre 1922 et ses attributions furent temporairement assignées à un commissaire extraordinaire qui, en deux années à peu près, mena à terme l'opération de redressement de l'entreprise : au 30 juin 1922 il y avait 226907 cheminots ; deux ans après il n'en restait que 174140 .

Les nombreux licenciements, effectués pour leur majeure partie en application de critères politiques, eurent pour effet une diminution du nombre des cheminots incroyable pour l'époque, à un moment où les chemins de fer n'étaient pas mis en question et où l'on considérait qu'ils devaient connaitre un développement sans fin.

Les cheminots reçurent un coup sévère et pour la première fois leur orgueil professionnel en souffrit, car ils étaient habitués à un emploi assuré et à bénéficier de la protection de leur syndicat. Toutefois, le prestige de la corporation ne fut pas atteint : pendant les vingt années que dura le fascisme, si les rythmes de travail s'intensifièrent les salaires restèrent élevés et les chemins de fer furent tenus en grande considération, par l'effet de la propagande qui mettait en avant la ponctualité des trains et grâce au progrès technique apporté par l'électrification des voies ferrées, pour laquelle l'Italie était à l'avant-garde mondiale. En revanche, après le fascisme les chemins de fer commencèrent à perdre du terrain dans le marché des transports et leur part se réduisit de façon préoccupante au profit de l'automobile et du transport routier.

Cependant, pendant les années 1950, le train était encore le moyen de transport fondamental ; il faisait partie de la vie quotidienne et de l'imaginaire de la plupart des Italiens, soit qu'il assure leurs déplacements quotidiens autour des métropoles, soit qu'il représente le moyen des grandes migrations qui partirent des campagnes du sud de l'Italie en direction des usines du Nord, caractéristiques du «miracle économique » (1953-1962). Pendant cette période deux films importants furent tournés qui contribuèrent à mieux faire connaittre la figure du cheminot : Destinazione Piovarolo (1953) et Il ferroviere (1955). 
Le premier, interprété par le célèbre acteur comique Totò, racontait l'histoire d'un chef de gare, Antonio La Quaglia, relégué pendant toute sa carrière professionnelle dans la petite gare de Piovarolo, où s'arrêtait seulement un train « acceléré » par jour. La Quaglia demandait continuellement sa mutation mais il n'arrivait pas à l'obtenir à cause de sa faible compétence et par manque d'appuis politiques, jusqu'au moment où il bloqua un convoi dans lequel un ministre des Transports « étourdi » voyageait : ce dernier lui promit de s'intéresser à son sort. Le film est une histoire comique pleine de brio qui fait largement appel à la satire politique, mais il montre aussi l'évolution du monde ferroviaire qui passe de la vapeur à l'électricité, l'utilisation du télégraphe et du téléphone et l'évolution des uniformes pendant la première moitié des années 1950.

Ilferroviere en revanche, tourné et interprété par Pietro Germi, est très différent; ce film raconte avec réalisme et par la voix et à travers les méditations d'un enfant, le fils du mécanicien Andrea Marcocci, le monde des chemins de fer et les problèmes sociaux qui étaient ceux des années 1950. Andrea conduit des trains express Rome-Milan. Le film reproduit le milieu de travail, syndical et les loisirs du personnel de conduite avec une minutie exceptionnelle ; l'histoire qu'il raconte occupe une année durant laquelle un accident de chemin de fer est évité de justesse. Une enquête est menée contre Marcocci qui l'accuse d'alcoolisme : il est destitué et doit travailler comme manœuvre ; à la fin du film il est accusé d'être un briseur de grève.

Pendant les années 1960 et 1970 le cheminot perdit peu à peu le prestige de son statut. Ce sont d'autres secteurs comme la métallurgie, l'industrie chimique ou le secteur tertiaire en constante augmentation, qui attiraient ouvriers et employés.

À la suite d'échecs dans l'adoption de nouvelles techniques, puis de nouvelles réductions du temps de travail, le nombre des employés des FS augmenta jusqu'à dépasser à nouveau les 220000 unités, comme au début des années 1920, et l'entreprise ferroviaire fut communément considérée comme un "réservoir de main-d'œuvre » destiné à réduire le chômage qui était préoccupant dans certaines régions du pays, surtout dans le Sud. C'est pour cette raison que la privatisation qui fut réalisée à partir des années 1980 a été traumatisante : cette restructuration industrielle fut la plus importante jamais mise en œuvre en Italie. En 1987 il y avait 220000 cheminots, il en reste aujourd'hui moins de 100 000, répartis entre les différentes sociétés qui composent les chemins de fer d'Italie (FS), transformée en société par actions dès 1992. 
La rapide réduction du personnel a créé des situations paradoxales; des cheminots prirent leur retraite à 40 ans grâce à des primes entièrement financées par l'État italien ${ }^{18}$, juste au moment où des coupes furent opérées dans les dépenses publiques pour respecter les critères européens de convergence ; paradoxalement des mises à la retraite furent bloquées et d'autres travailleurs que les cheminots ne parvinrent pas à obtenir leur pension.

La privatisation suscita des situations conflictuelles à l'intérieur de l'entreprise elle-même, des grèves et les cheminots occupèrent à nouveau le devant da la scène. Les mécaniciens surtout firent continuellement grève et encouragèrent une nouvelle forme de syndicat, les comitati di base qui s'opposaient aux syndicats traditionnels; ils commencèrent à lutter pour l'augmentation des salaires et parvinrent à obtenir des résultats même s'ils payèrent cher leur victoire, étant donné le nombre désormais limité des cheminots.

Pendant les années qui viennent de s'écouler, la restructuration a été caractérisée par l'introduction rapide des nouvelles technologies (matériel roulant moderne, régulation centralisée du trafic, automatisation des passages à niveau) et par l'embauche de nombreux jeunes gens dans le cadre de contrats qui ne donnent pas à leur emploi le caractère traditionnel de stabilité du travail ferroviaire. La vieille identité professionnelle a donc été bouleversée par une façon de travailler complètement différente.

Cependant le passé avait déjà connu des transformations de grande ampleur. Il faut prendre en compte non seulement la restructuration intervenue pendant le fascisme mais aussi le passage de la vapeur à l'électricité et au Diesel, la fin des postes de garde le long des voies ferrées, le passage du télégraphe au téléphone, les réductions du temps de travail. Toutefois la dernière mutation en date, parce qu'elle a été concentrée en un temps très court, est certainement la plus grande que les cheminots italiens aient vécu dans leur histoire (fig. 4).

18- Loi du 7 juin $1990 \mathrm{n}^{\circ} 141$. 


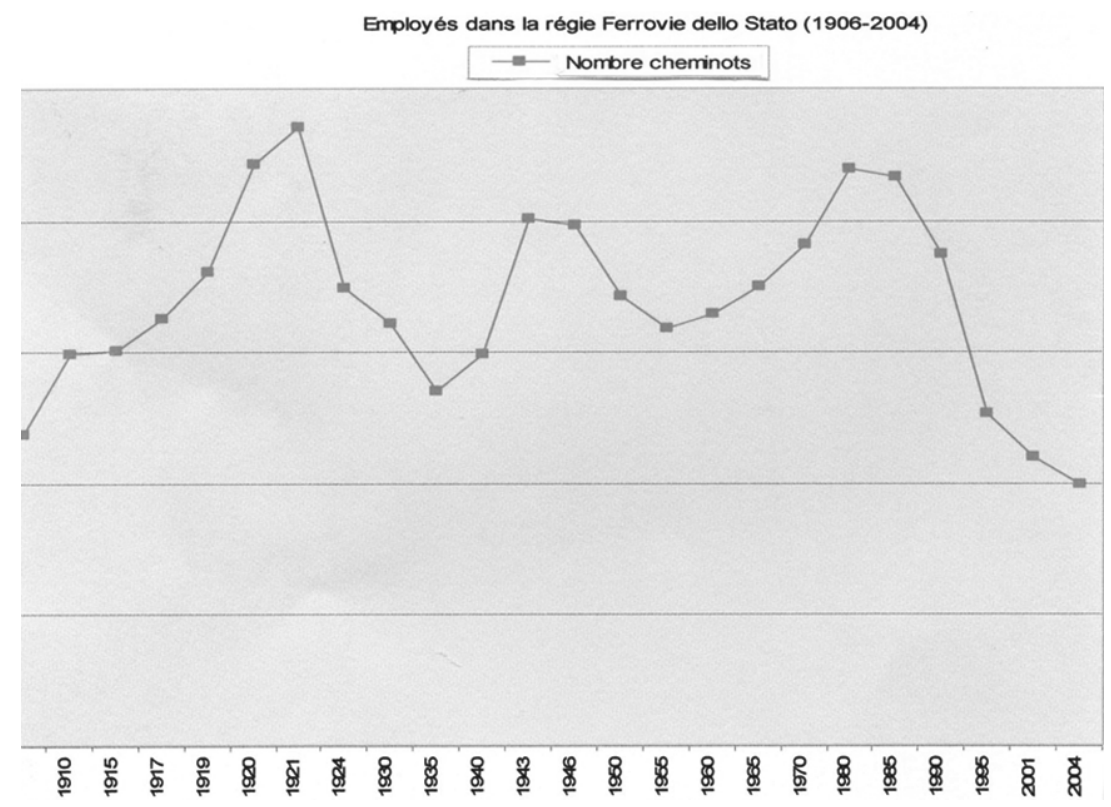

4 Évolution des effectifs des agents des Ferrovie dello Stato (1906-2004).

Le personnel a été réduit de moitié, les jolies gares de campagne sont devenues des points d'arrêt anonymes sans jardins, sans guichets, sans toilettes. Elles sont devenues des proies faciles pour les vandales. Les trains à grande vitesse sont arrivés (le Pendolino et l'ETR 500), suivant l'exemple du TGV français, mais on n'a pas construit des voies nouvelles dédiées pour les faire rouler : les lignes à grande vitesse projetées entre Turin et Venise et entre Milan et Naples doivent encore être achevées ; en 2006 seuls les tronçons entre Rome et Naples et entre Turin et Novare ont été mis en service.

Les nouvelles technologies ont permis l'usage de la télécommande des aiguilles et des signaux pour des itinéraires très longs et ont rendu superflu le personnel de gare ; les chemins de fer secondaires ont été entre-temps abandonnés à leur destin sans aucune possibilité de valorisation.

Pendant ce tournant historique la profession de cheminot a perdu la plupart de son charme. Les figures romantiques comme les chefs de train et les chefs de gare sont en train de disparaitre ; les chemins de fer sont aujourd'hui un mécanisme électronique complexe beaucoup plus impersonnel qu'autrefois et sans la passion syndicale, politique et culturelle qui avait caractérisé jusqu'alors le monde des trains. 